\title{
Nonlinear Thermodynamic Model of Boundary Friction
}

\author{
I. A. Lyashenko ${ }^{a,}$, A. V. Khomenko ${ }^{a}$, and L. S. Metlov ${ }^{b}$ \\ ${ }^{a}$ Sumy State University, ul. Rimskogo-Korsakova 2, Sumy, 40007 Ukraine \\ *e-mail:nabla04@ukr.net \\ ${ }^{b}$ Donetsk Institute for Physics and Engineering named after A.A. Galkin, National Academy of Sciences of Ukraine, \\ ul. Rozy L'uksemburg 72, Donetsk, 83114 Ukraine
}

Received June 29, 2010

\begin{abstract}
Melting of an ultrathin lubricant film confined between two atomically flat surfaces is studied. An excess volume parameter is introduced, the value of which is related to the presence of defects and inhomogeneities in the lubricant. Via minimization of the free energy, the Landau-Khalatnikov kinetic equation is obtained for this parameter. The kinetic equation is also used for relaxation of elastic strains, which in its explicit form contains the relative shear velocity of the rubbing surfaces. With the numerical solution of these equations, a phase diagram with domains corresponding to the sliding and dry stationary friction regimes is built at a fixed shear velocity. A simple tribological system is used to demonstrate that in the dynamic case, three friction regimes can occur, namely, dry, stick-slip, and sliding friction. It is shown that a lubricant can melt when the shear velocity exceeds a critical value and with elevation of its temperature. The dependence of the dynamic friction force on the pressure applied to the surfaces, the temperature of the lubricant, and the shear velocity is considered. It is shown that growth of pressure leads to the forced ordering and solidification of the lubricant.
\end{abstract}

Keywords: lubricant, friction force, shear stresses and strains, free energy, phase transition, stick-slip regime.

DOI: $10.3103 / \mathrm{S} 1068366611020061$

\section{INTRODUCTION}

Recently, boundary friction conditions have been actively studied in connection with increasing application of nanosized systems. These conditions occur for lubricating layer thicknesses of $<10$ atomic diameters $[1,2]$. Experiments show that a thin lubricating layer demonstrates abnormal properties relative to volumetric ones [3]. In particular, stick-slip motion is observed $[3,4]$, which commonly characterizes dry friction. The regime is attributed to solidification of the lubricant due to its confinement between two rubbing surfaces and its subsequent stick-slip melting when shear stresses exceed the yield point ("shear melting").

In order to reduce the contribution of irregularities, atomically flat mica surfaces [5-7], quartz [8], and sapphire [9] are commonly used in such experiments. Polymer chains [10], surfactants [11, 12], protein compositions [13], and even metallic layers deposited on mica [14] can be used as lubricants.

There are several phenomenological models that allow one to explain in part the results observed experimentally, e.g., thermodynamic [15], mechanistic $[16]$, and synergetic $[17,18]$ models. They are of deterministic $[16,17]$ and stochastic $[19,20]$ natures. In addition, methods of molecular dynamics are employed in investigations [21, 22]. Lubricants are shown to provide several kinetic regimes, between which transitions occur that lead to stick-slip friction [3]. These transitions are represented as first-order phase transitions [23] between states that are not stable thermodynamic phases but represent kinetic friction regimes. The authors of work [19] have identified three main regimes, namely, sliding at low shear velocities, the regular stick-slip regime, and sliding at high shear velocities. These data are substantiated by numerous experiments $[1,3,4,8]$.

An approach has been developed elsewhere [17, 18] according to which transition of the lubricant from the solid to liquid state results from thermodynamic and shear melting. The processes are shown to be due to self-organization of the fields of shear stress and strain, as well as lubricant temperature, with account of additive noise of the above values [24-27] and correlated temperature fluctuations [28]. Hysteresis behavior observed experimentally [29-31] is considered in works [32-34]. In work [35], within the framework of the above-mentioned model, the periodic stick-slip friction regime is discussed; this regime, however, has a stochastic component and can occur only in the presence of fluctuations in the system. A disadvantage of this model is that it does not account for the load applied to the friction surfaces and uses approximations when deriving the basis equations [17]. 
The present work proposes a model based on the expansion of free energy by the power of the parameter of excess volume arising from the formation of the defect structure in a lubricant material during its melting. The liquid state is commonly interpreted as the region of plastic flow on the loading diagram and characterized by the defects present in the lubricant [15]. In this paper the authors use an approach based on the Landau theory of phase transitions [36-39] to describe strongly nonequilibrium processes occurring during the sliding of two solids separated by a lubricant layer.

\section{BASIC EQUATIONS}

Melting of a lubricating film $<10$ molecular layers thick is determined by increasing volume [21] and diffusion coefficient $[21,22,40,41]$. Since of these two values, the volume is the parameter experimentally observed, in order to describe the lubricant state, we will introduce a parameter $f$ as an excess volume arising from chaotization of the solid structure in the course of melting. With increasing $f$, the density of defects in the lubricant rises and owing to their transportation under the action of applied stresses, it transfers to the kinetic regime of plastic flow (the liquid phase).

We write the function of the density of free energy $\Phi$ versus excess volume in the form of expansion in terms of the parameter $f$ in the form of

$$
\Phi=\Phi_{0}+\frac{c}{2}(\nabla f)^{2}-\phi_{0} f+\frac{1}{2} \phi_{1} f^{2}-\frac{1}{3} \phi_{2} f^{3}+\frac{1}{4} \phi_{3} f^{4},
$$

where $\Phi_{0}, c, \phi_{0}, \phi_{1}, \phi_{2}$, and $\phi_{3}$ are expansion constants.

We will take the dependence on the invariants of elastic strain $\varepsilon_{i j}^{e}$ and lubricant temperature into account only at smaller expansion degrees:

$$
\begin{gathered}
\Phi_{0}=\Phi_{0}^{*}+\frac{1}{2} \lambda\left(\varepsilon_{i i}^{e}\right)^{2}+\mu\left(\varepsilon_{i j}^{e}\right)^{2} ; \\
\phi_{0}=\phi_{0}^{*}+\frac{1}{2} \bar{\lambda}\left(\varepsilon_{i i}^{e}\right)^{2}+\bar{\mu}\left(\varepsilon_{i j}^{e}\right)^{2}+\alpha T .
\end{gathered}
$$

The first invariant is the trace of the strain tensor $\varepsilon_{i i}^{e}=\varepsilon_{1}^{e}+\varepsilon_{2}^{e}+\varepsilon_{3}^{e}$ and the second is determined by the following equation [42]:

$$
\begin{gathered}
\left(\varepsilon_{i j}^{e}\right)^{2} \equiv\left(\varepsilon_{i i}^{e}\right)^{2}-2 I_{2}=\left(\varepsilon_{1}^{e}+\varepsilon_{2}^{e}+\varepsilon_{3}^{e}\right)^{2} \\
-2\left(\varepsilon_{1}^{e} \varepsilon_{2}^{e}+\varepsilon_{1}^{e} \varepsilon_{3}^{e}+\varepsilon_{2}^{e} \varepsilon_{3}^{e}\right)=\left(\varepsilon_{1}^{e}\right)^{2}+\left(\varepsilon_{2}^{e}\right)^{2}+\left(\varepsilon_{3}^{e}\right)^{2} .
\end{gathered}
$$

According to (1), the elastic stresses arising in the lubricant are found as

$$
\sigma_{i j}^{e}=\frac{\partial \Phi}{\partial \varepsilon_{i j}^{e}}=\lambda \varepsilon_{i i}^{e} \delta_{i j}+2 \mu \varepsilon_{i j}^{e}-\left(\bar{\lambda} \varepsilon_{i i}^{e} \delta_{i j}+2 \bar{\mu} \varepsilon_{i j}^{e}\right) f .
$$

Expression (4) can be presented in the form of the Hooke effective law [43]

$$
\sigma_{i j}^{e}=2 \mu_{e f f} \varepsilon_{i j}^{e}+\lambda_{e f f} \varepsilon_{i i}^{e} \delta_{i j}
$$

with effective elastic parameters

$$
\begin{aligned}
& \mu_{e f f}=\mu-\bar{\mu} f ; \\
& \lambda_{\text {eff }}=\lambda-\bar{\lambda} f,
\end{aligned}
$$

which decrease with melting and increasing excess volume $f$.

It is easy to show that (see the Appendix)

$$
\begin{gathered}
\varepsilon_{i i}^{e}=\frac{n}{\lambda_{e f f}+\mu_{\text {eff }}} ; \\
\left(\varepsilon_{i j}^{e}\right)^{2}=\frac{1}{2}\left[\left(\frac{\tau}{\mu_{e f f}}\right)^{2}+\left(\varepsilon_{i i}^{e}\right)^{2}\right],
\end{gathered}
$$

where $n$ and $\tau$ are the normal and tangential components of the stresses acting on lubricant from the side of rubbing surfaces. ${ }^{2}$

Relationships (8) and (9) show the relation between the tensor components and their invariants in the linear theory of elasticity [42].

We write the equation for the nonequilibrium parameter $f$ in the form of the Landau-Khalatnikov equation

$$
\dot{\tau} \dot{f}=-\frac{\partial \Phi}{\partial f},
$$

where the time of relaxation $\tau_{f}$ is introduced. In its explicit form it looks like

$$
\begin{gathered}
\tau_{f} \frac{\partial f}{\partial t} \\
=-c \nabla^{2} f+\phi_{0}-\phi_{1} f+\phi_{2} f^{2}-\phi_{3} f^{3}-\frac{n^{2}(\bar{\lambda}+\bar{\mu})}{\left(\lambda_{e f f}+\mu_{e f f}\right)^{2}},
\end{gathered}
$$

where the appearance of the last component is due to the fact that invariants (8) and (9) depend on the parameter $f$.

We obtain an equation that determines the relative shear velocity of the rubbing surfaces $V_{i j}$ and elastic strains $\varepsilon_{i j}^{e}$ arising in the lubricant. For this purpose, we

\footnotetext{
${ }^{1}$ At $f>\mu / \bar{\mu}$ it should be taken that $\mu_{\text {eff }}=0$ and at $f>\lambda / \bar{\lambda}$ it should be taken that $\lambda_{\text {eff }}=0$.

${ }^{2}$ Shear stress $\tau$ is found from expression (5) at $i \neq j$; i.e., $\delta_{i j}=0$. At $\mu_{\text {eff }}=0$, the component $\tau / \mu_{\text {eff }}$ in (9) should be replaced, in line with (5), by $2 \varepsilon_{i j}^{e}$.
} 
use the Debye approximation relating the elastic and the plastic strain $\varepsilon_{i j}^{p l}[15]$ :

$$
\dot{\varepsilon}_{i j}^{p l}=\frac{\varepsilon_{i j}^{e}}{\tau_{\varepsilon}},
$$

where $\tau_{\varepsilon}$ is the Maxwell time of relaxation of internal stresses. Full strain in the layer will be found as

$$
\varepsilon_{i j}=\varepsilon_{i j}^{e}+\varepsilon_{i j}^{p l}
$$

This strain specifies the velocity of the upper block according to the relationship [44]

$$
V_{i j}=h \dot{\varepsilon}_{i j}=h\left(\dot{\varepsilon}_{i j}^{e}+\dot{\varepsilon}_{i j}^{p l}\right) \text {, }
$$

where $h$ is the thickness of the lubricant film. The expression for the elastic component of shear strain can be derived from the three above-mentioned relationships

$$
\tau_{\varepsilon} \dot{\varepsilon}_{i j}^{e}=-\varepsilon_{i j}^{e}+\frac{V_{i j} \tau_{\varepsilon}}{h}
$$

For simplicity, the present work considers a homogeneous system and in relationships (1) and (11), it is taken that $\nabla \equiv 0$.

\section{THERMODYNAMIC AND SHEAR MELTING}

The set of kinetic equations (11) and (15) with account for definitions (2), (5)-(9) is closed and can be used to study the kinetics of lubricant melting. In this section we consider stationary friction regimes that result from system evolution. According to Eq. (15) the elastic component of shear strain becomes stationary with time

$$
\varepsilon_{i j 0}^{e}=\frac{V_{i j} \tau_{\varepsilon}}{h} .
$$

In order to determine the stationary states of all values, it is necessary to solve evolution equation (11) using (2), (5) - (9) and finding the strain value from (16).

In experiments, atomically flat mica surfaces are often used as friction surfaces and quasispherical molecules of octamethylcyclotetrasiloxane (OMCTS) and linear chain molecules of tetradecane or hexadecane $[3,31]$, as lubricants. The above-mentioned experiments are carried out under the following conditions: thickness of the lubricant layer $h \approx 10^{-9} \mathrm{~m}$, contact area $A \approx 3 \times 10^{-9} \mathrm{~m}^{2}$, load to the upper friction surface $L=(2-60) \times 10^{-3} \mathrm{~N}$, which corresponds to normal stresses $n=-L / A=-(6.67-200) \times 10^{5} \mathrm{~Pa}$.

Here, the friction force is $F \approx(2-40) \times 10^{-3} \mathrm{~N}$. Within the framework of the above-mentioned experimental works, it has been found that the lubricant melts if the temperature exceeds a critical value $T>T_{c 0} \approx 300 \mathrm{~K}$ or at a shear velocity $V>V_{c 0} \approx 400 \mathrm{~nm} / \mathrm{s}$.

These values can vary depending on the lubricant used and the experiment geometry.
In the studied model, the following values for the constants of the theory are chosen in line with the experimental data: $\Phi_{0}^{*}=20 \mathrm{~J} / \mathrm{m}^{3} ; \lambda=2 \times 10^{11} \mathrm{~Pa}$; $\bar{\lambda}=10^{8} \mathrm{~Pa} ; \mu=4.1 \times 10^{11} \mathrm{~Pa} ; \bar{\mu}=4 \times 10^{11} \mathrm{~Pa} ; \phi_{0}^{*}=$ $5 \mathrm{~J} / \mathrm{m}^{3} ; \phi_{1}=1100 \mathrm{~J} / \mathrm{m}^{3} ; \phi_{2}=2700 \mathrm{~J} / \mathrm{m}^{3} ; \phi_{3}=2070 \mathrm{~J} / \mathrm{m}^{3} ;$ $\alpha=0.45 \mathrm{~J} \mathrm{~K}^{-1} / \mathrm{m}^{3} ; h=10^{-9} \mathrm{~m} ; \tau_{f}=1$ Pa s; $\tau_{\varepsilon}=10^{-8} \mathrm{~s} ;$ $n=-7 \times 10^{5} \mathrm{~Pa}$.

Note that the time of relaxation of excess volume $\tau_{f}$ has a viscosity dimension. In fact, this means that the time needed for the stationary regime to be set increases with increasing efficient viscosity of the lubricant.

At a zero shear velocity (shear stresses and strains are zero) and a temperature below the critical value $T<T_{c 0}$ the lubricant is in the solid phase and the excess volume takes a small value that increases with increasing lubricant temperature (the dot-and-dash portion of the curve). At the temperature $T=T_{c 0}$ the excess volume increases jumpwise and the lubricant transforms into the liquid state (the continuous portion of the curve). With further elevation of the temperature it solidifies at lower $T=T_{c}^{0}$. The dependence has the hysteresis nature, which corresponds to first-order phase transitions. According to Fig. 1a, with increasing shear velocity the lubricant is melted at a lower temperature. At a temperature higher than its critical value, the lubricant is always liquid (curve 4) irrespective of temperature, and its full melting occurs owing to shear.

Figure 2a shows the dependence of the density of free energy $F$ on $f$ at the parameters of curve 1 in Fig. 1a. At low temperatures (the upper curve), one potential minimum is implemented at small $f$ (solid lubricant) that corresponds to the dot-and-dash curve in Fig. 1a. With increasing temperature (the middle curve), an additional minimum appears, corresponding to the continuous curve in Fig. 1a. However, the system cannot transform to the proper state since it is separated from the first minimum by an energy maximum (dashed curve in Fig. 1a). With further increase in $T$, the separating maximum disappears (the lower curve) and the lubricant transforms sharply, following the mechanism of phase transition, to the state that corresponds to the potential minimum at a larger excess volume; i.e., it melts. If now the temperature drops, then with the appearance of a minimum at small $f$, the system cannot again transform sharply into the corresponding state because of the separating maximum. With its disappearance at $T=T_{c}^{0}$, the lubricant solidifies in a jumpwise manner.

If the lubricant temperature is fixed, then when the shear velocity exceeds its critical value $V_{c 0}$, the lubricant melts and when $V_{i j}<V_{c}^{0}$, the lubricant solidifies (Fig. 1b). Here, the situation resembles the behavior of the system under rising temperature (Fig. 1a), yet 

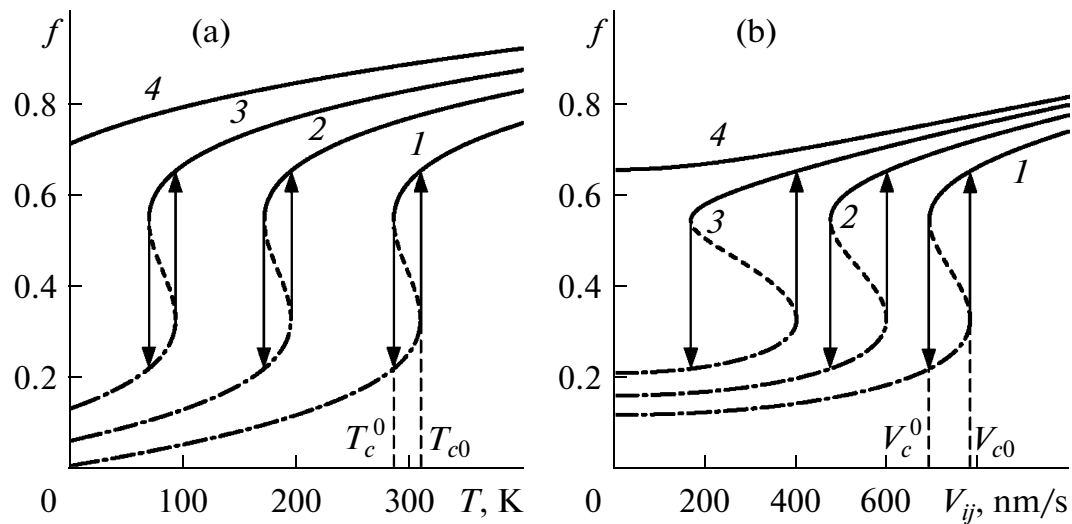

Fig. 1. Stationary excess volume (dimensionless value) versus lubricant temperature (a) and shear velocity (b): a-curves $1-4$ correspond to the fixed values of shear velocity $V_{i j}=0,800,1100$, and $1400 \mathrm{~nm} / \mathrm{s} ; \mathrm{b}$ - curves $1-4$ are built at fixed temperatures $T=$ $200,245,280$, and $310 \mathrm{~K}$.
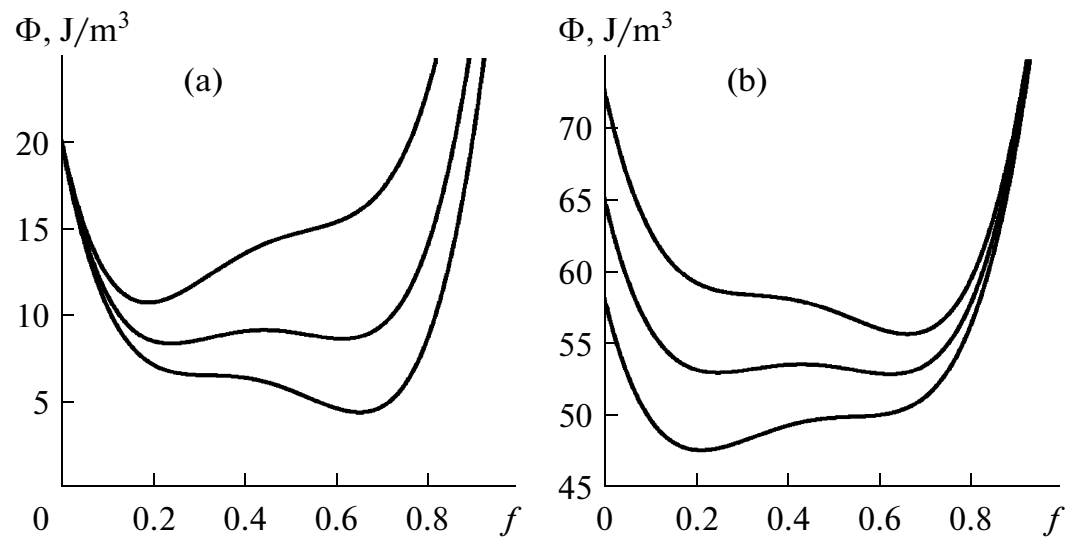

Fig. 2. Density of free energy versus parameter $f$ (dimensionless value): a-curves located from the top to down correspond to $T=270,295$, and $310 \mathrm{~K}$ and zero shear velocity; b-curves located from bottom to top correspond to $V_{i j}=680,740$, and $800 \mathrm{~nm} / \mathrm{s}$ and $T=200 \mathrm{~K}$.

the difference is that in the case of shear melting the area restricted by hysteresis increases with increasing temperature. The lubricant melts at lower shear velocities with increasing temperature. Figure $2 b$ shows the profile of free energy at the parameters of curve 1 in Fig. 1b, which is similar to the profile of energy when the lubricant melts under temperature elevation.

Figure $3 \mathrm{a}$ shows the dependence of the critical shear velocities for lubricant melting $V_{c 0}$ and solidification $V_{c}^{0}$ on temperature $T$. Thus, above the curve $V_{c 0}$ the lubricant is in the liquid state and the sliding friction regime occurs ( $S F$ region). At shear velocities below $V_{c}^{0}$ the solid state of the lubricant occurs. Between the curves in Fig. 3a, the hysteresis region arises shown in Fig. 1, which corresponds to the potential shown by the middle curve in Fig. 2. Thus, the lubricant state is here undetermined and depends on the initial conditions. According to Fig. 3a, both critical regions decrease with temperature elevation until they become zero and full thermodynamic melt- ing occurs. Note that the lubricant melts even at zero temperature $T$ as the shear velocity surpasses its critical value (the boundary of the $S F$ region on the $Y$-axis). In other words, Fig. 3a is a phase diagram with two stationary friction regimes. The distance between the curves in the figure in the horizontal direction at a constant velocity (segment 1 ) is the hysteresis width that is implemented in Fig. 1a and the distance in the vertical direction at $T=$ const (segment 2 ) specifies the width of the hysteresis in Fig. 1b. As follows from Fig. 3a, the width of the temperature hysteresis (segment 1) remains constant with varying velocity whereas the velocity hysteresis (segment 2) becomes considerably wider with increasing temperature compared to low temperatures. This fact is represented in Fig. 1. Note that Fig. 3a can also be interpreted as the dependence of critical temperatures $T_{c 0}$ and $T_{c}^{0}$ on the shear velocity $V_{i j}$.

Figure $3 \mathrm{~b}$ demonstrates the temperature dependence of the hysteresis (Fig. 1b) width. The above- 

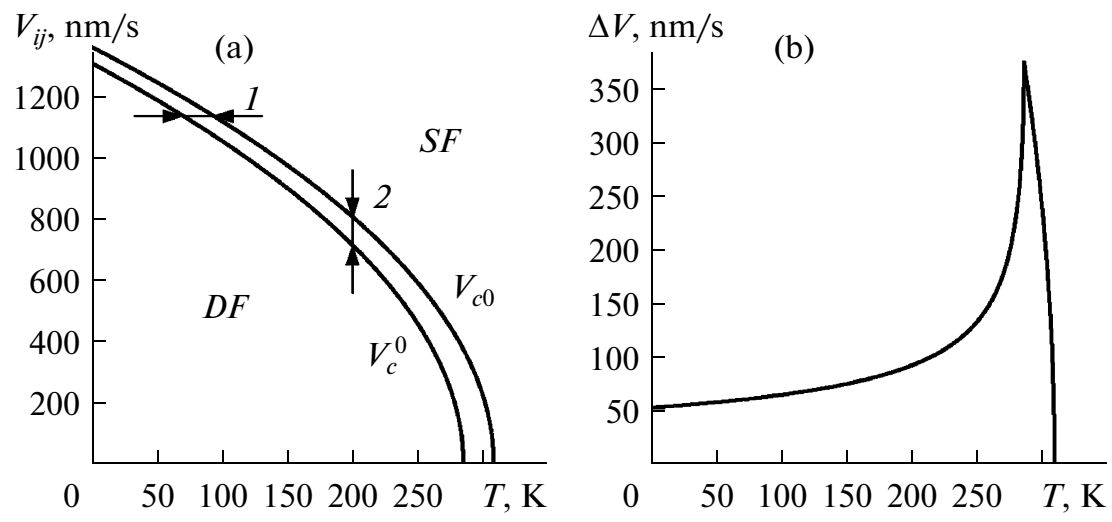

Fig. 3. Phase diagram with the regions of sliding $(S F)$ and dry $(D F)$ friction (a) and hysteresis width versus lubricant temperature (b).

mentioned dependence is obtained by moving segment 2 (Fig. 3a) along the $T$-axis. The width of the hysteresis loop increases within almost the entire temperature range. Its sharp decrease down to zero is due to the fact that, according to Fig. 3a, critical velocity $V_{c}^{0}$ becomes zero at lower temperatures than $V_{c 0}$ and after this moment $V_{c}^{0}$ should be taken as zero. Therefore, the peak in Fig. $3 \mathrm{~b}$ corresponds to the temperature at which the shear velocity $V_{c}^{0}$ becomes zero. At this temperature the lubricant cannot solidify due to the decreasing shear velocity (according to Fig. 3a, at $V_{c}^{0}=0$ the system cannot fall within the $D F$ region, however, the lubricant can initially fall within the intermediate region and be solidlike at the same time up to the velocity $V_{i j}<V_{c 0}$ ). The temperature (Fig. 3b) at which the hysteresis width becomes zero conforms to the situation when the lubricant is liquid at any shear velocity (according to Fig. 3a, in this case the system is always within the $S F$ region of sliding friction). The calculation of the dependence of hysteresis width at $\Delta T=T_{c 0}-T_{c}^{0}$ on the shear velocity $V_{i j}$ shows that it remains constant at all velocities and $\Delta T \approx$ $23.426 \mathrm{~K}$, except for the velocities at which temperature $T_{c}^{0}$ is zero. Here, as in the case shown in Fig. 3b, the hysteresis width becomes zero monotonously with increasing shear velocity (see Fig. 3a).

\section{FRICTION FORCE}

Experimental works often discuss the kinetic dependences of the friction force at various shear velocities, lubricant layer thicknesses, and normal pressure [1, 3, 4, 29-31]. We analyze the influence of the friction force on lubricant temperature, shear velocity, and pressure.
The full pressure arising within the lubricant layer is determined as the sum of the elastic $\sigma_{i j}^{e}$ and viscous $\sigma_{i j}^{v i s c}$ components:

$$
\sigma_{i j}=\sigma_{i j}^{e}+\sigma_{i j}^{v i s c} \text {. }
$$

The friction force is found in a standard way:

$$
F_{i j}=\sigma_{i j} A \text {, }
$$

where $A$ is the area of the contact surfaces. Viscous stresses can be calculated according to the empirical formula [44]

$$
\sigma_{i j}^{v i s c}=\frac{\eta_{e f f} V_{i j}}{h},
$$

where $\eta_{\text {eff }}$ is the effective viscosity of the lubricant. The boundary lubricant is a non-Newtonian liquid and commonly has the complex dependence $\eta_{\text {eff }}(\dot{\varepsilon})$. By way of example, the viscosity of polymer solutions and melts commonly decrease with increasing strain $\eta(\dot{\varepsilon})$ (pseudoplastic liquids); in the case of suspensions of solid particles the viscosity increases with increasing $\dot{\varepsilon}$ (dilatants). Therefore, for qualitative analysis we use the simple approximation [44]

$$
\eta_{\text {eff }}=k\left(\dot{\varepsilon}_{i j}\right)^{\gamma}
$$

allowing us to take both of the above-mentioned situations into account.

Here the proportionality coefficient $k\left(\mathrm{~Pa} \cdot \mathrm{s}^{\gamma+1}\right)$ is introduced. According to (20), in pseudoplastic liquids $\gamma<0$, in dilatants $\gamma>0$, and in non-Newtonian liquids $\gamma=0$ since, in line with (20), in this case the viscosity is independent of the strain rate.

With regard to (14) and (20) the expression for viscous stresses is written as follows:

$$
\sigma_{i j}^{v i s c}=k\left(\frac{V_{i j}}{h}\right)^{\gamma+1} \text {. }
$$



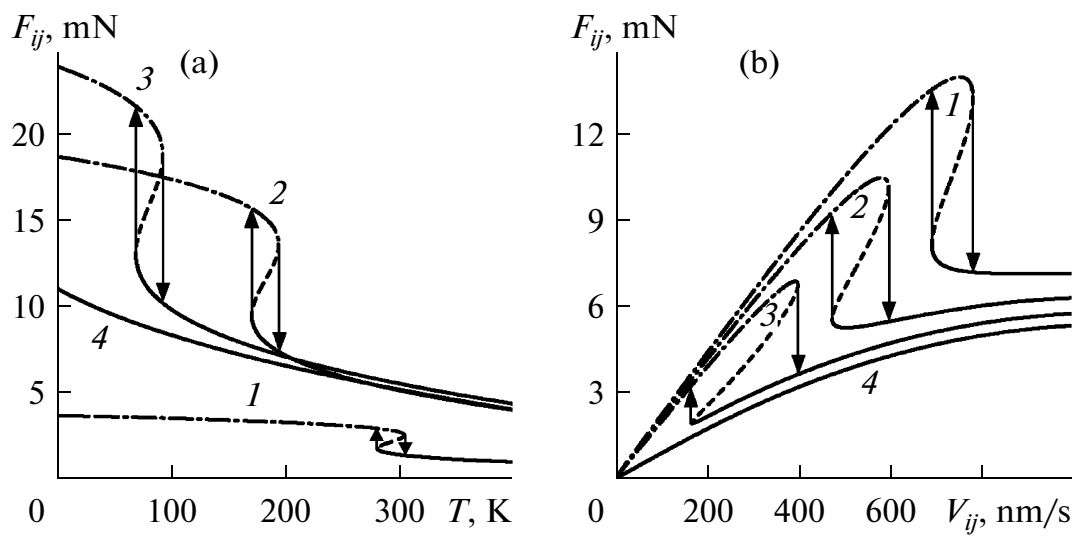

Fig. 4. Friction force versus temperature (a) and shear velocity (b) at $\gamma=2 / 3, k=1 \mathrm{~Pa} \cdot \mathrm{s}^{5 / 3}$ and contact area $A=3 \times 10^{-9} \mathrm{~m}^{2}$ : curves $1-4$ conform to constant shear velocities $V_{i f}=150,800,1100$, and $1400 \mathrm{~nm} / \mathrm{s} ; \mathrm{b}$ - curves $1-4$ correspond to fixed temperatures $T=200,245,280$, and $310 \mathrm{~K}$.

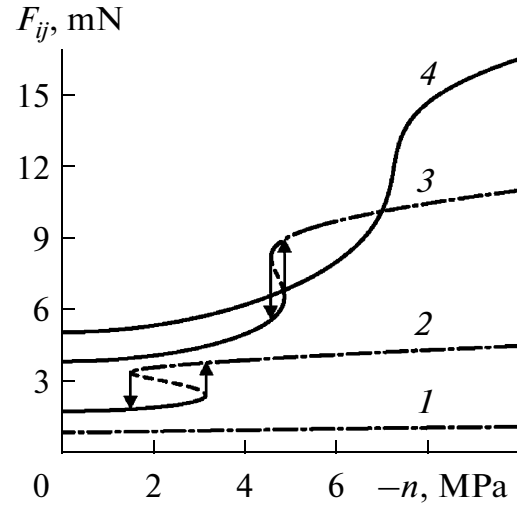

Fig. 5. Friction force versus normal pressure on the friction surface at the parameters of Fig. 4 and $T=305 \mathrm{~K}$. Curves $1-4$ conform to constant shear velocities $V_{i j}=50$, 200,500 , and $800 \mathrm{~nm} / \mathrm{s}$

On substituting (17) and (21) into (18) we obtain the final expression for the friction force ${ }^{3}$

$$
F_{i j}=\left[\sigma_{i j}^{e}+k \operatorname{sgn}\left(V_{i j}\right)\left(\frac{\left|V_{i j}\right|}{h}\right)^{\gamma+1}\right] A,
$$

where $\sigma_{i j}^{e}$ is specified by formula (5) at $i \neq j$.

Dependence (22) is shown in Fig. 4. All curves in Fig. 4a correspond to the curve parameters (Fig. 1a) except for curve 1 .

This is due to the fact that in Fig. 1a the first curve is constructed at zero shear velocity and during rest the friction force is zero. All curves in Fig. $4 b$ correspond to the curve parameters in Fig. 1b. In line with Fig. 4a, the friction force decreases with rising temperature. This is due to increasing $f$, with which the effective

\footnotetext{
${ }^{3}$ Here the sign function $\operatorname{sgn}(x)$ and absolute shear velocity $\left|V_{i j}\right|$, are introduced since the velocity can take negative values as well.
}

shear module $2 \mu_{\text {eff }}(6)$ decreases, which results in a lower elastic component of shear stresses (5) and, correspondingly, a lower friction force (22). On the plot one can observe a hysteresis since in the phase transition the shear module (6) varies stepwise. At the parameters of curve 4 the lubricant is always in the liquid state and the friction force decreases with temperature owing to reduced shear module (lubricant dilution). Figure $4 \mathrm{~b}$ demonstrates a somewhat different behavior of the lubricant. Here, according to (22), with low shear velocities, the lubricant in the solid state, and large $\sigma_{i j}^{e}$, dry friction occurs. An increase in the shear velocity under dry friction leads to increasing friction force (22). With further increase in the velocity, the lubricant is melted and the elastic shear stresses (5) decrease considerably, leading to a sharp decrease in the full friction force. With still further increase in the velocity, $F_{i j}$ increases.

According to curve 4, with the lubricant in the liquid state, the friction force (22) increases due to the increased shear velocity. Note that the results shown in Fig. $4 \mathrm{~b}$ conform qualitatively to the new friction map of the boundary conditions that was obtained in generalizing the experimental data from work [44].

Figure 5 presents the dependence $F_{i j}$ on the normal external stresses, the action of which is directed at confinement of the rubbing surfaces.

According to curve 1, at low shear velocities the lubricant is solid over the stress range chosen, which provides an increase in the friction force with increasing pressure; however, the growth is insignificant at the scale of the Figure. Curves 2 and 3 show that within the intermediate range of shear velocities the lubricant is liquid under small normal pressures and further confinement of the surfaces leads to lubricant solidification and increase in the overall friction force. At large shear velocities (curve 4) the first-order phase transition between liquid-like and solid-like lubricant struc- 


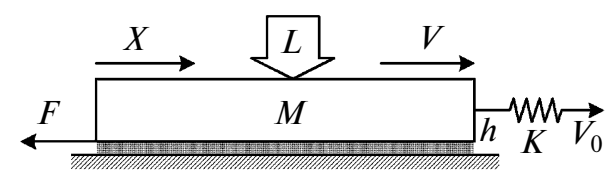

Fig. 6. Diagram of the tribosystem (designations are given in the text).

tures does not occur. Here, continuous transition (the region of inflection of curve 4) between the abovementioned states takes place. A similar dependence is discussed elsewhere [44].

\section{STICK-SLIP REGIME}

The dependences cited in the preceding sections of the paper are obtained at a fixed shear velocity of the upper rubbing surface. However, the dynamic characteristics of any tribological system are governed by the properties of the system as a whole. In particular, according to the experimental data, the stick-slip regime can occur within the region of hysteresis considered above [3, 16, 19-21, 31, 41, 44]. A typical diagram of the tribological system is shown in Fig. 6. Here, a spring with rigidity $K$ is connected to a block with mass $M$ to which a supplementary normal load $L$ is applied. The block is located on a flat surface and is separated from it by a lubricant layer of thickness $h$. The free end of the spring is put into motion at a constant velocity $V_{0}$. While the block moves, the friction force $F(22)$ arises, providing resistance to its movement. As for ultrathin lubricant layers under boundary friction, in the general case, the velocities $V$ of the block and $V_{0}$ of the spring do not coincide because of the oscillating nature of force $F$, which leads to stickslip motion.

The equation for the movement of the upper block has the following form $[3,15,16]^{4}$ :

$$
M \ddot{X}=K\left(\int_{0}^{t} V_{0} d t^{\prime}-X\right)-F,
$$

where $t=t^{\prime}$ is the time of movement of the free end of the spring. To calculate the time dependence of the friction force, Eq. (23) should be solved jointly with (11) and (15), determining $F$ from (22). However, owing to the thin lubricant layer, the time of strain relaxation $\tau_{\varepsilon}$ can be taken as small compared to the time of excess volume relaxation $\tau_{f}$. Therefore, within the framework of $\tau_{f} \gg \tau_{\varepsilon}$ we solve two Eqs. (23) and (11), jointly finding strain from (16). The result of the solution of these equations is shown in Fig. 7, according to which the friction force first increases since the

\footnotetext{
${ }^{4}$ Here, for convenience, tensor designations are omitted since we consider the shear in one direction.
}

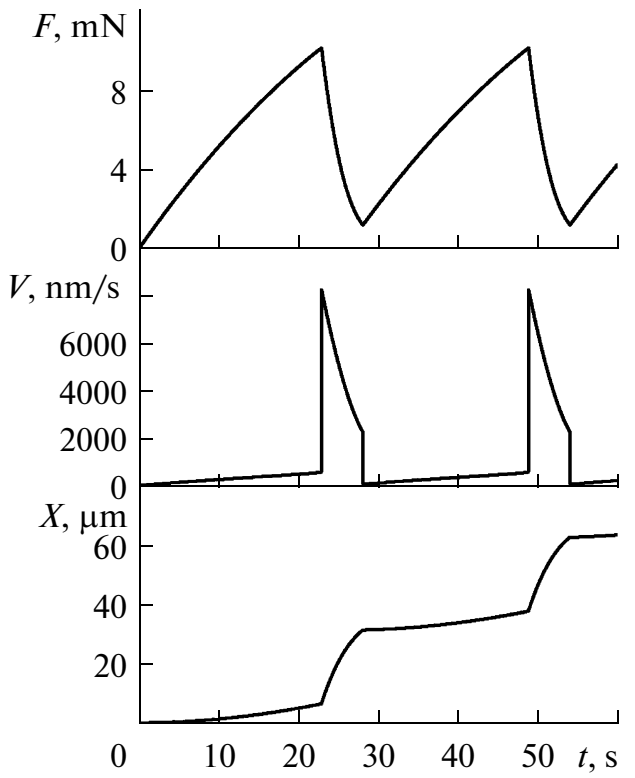

Fig. 7. Friction force $F$, shear velocity of the rubbing block $V$, and its coordinate $X$ versus time at parameters $n=-7 \times 10^{5} \mathrm{~Pa}$; $M=0.1 \mathrm{~kg} ; K=480 \mathrm{~N} / \mathrm{m} ; T=250 \mathrm{~K} ; V_{0}=1200 \mathrm{~nm} / \mathrm{s}$.

lubricant is in the solid state and the shear velocity $V$ increases.

When the velocity exceeds the critical $V_{c 0}$, the lubricant is melted, the slip velocity of the rubbing block increases and it quickly moves a large distance. Here, the spring tension, and correspondingly the shear velocity, decrease. When the velocity becomes twice as low as the value necessary to keep the lubricant in the liquid state, the lubricant solidifies and the friction force starts increasing again. The process outlined is repeated periodically with time. Note that the velocity at which the lubricant solidifies does not coincide with the similar velocity shown in Fig. 1 . This is related to the sharp increase in the shear velocity $V$ during melting and the corresponding increase in excess volume $f$. According to (6) the shear module becomes less than zero and it should be taken as zero, which distorts the view of potential (1). In this case, with the availability of elastic strains (16), the elastic stresses in the lubricant according to (5) are zero, which causes a decrease in the friction force while the lubricant flows.

Figure 8 shows the kinetic dependences of friction, excess volume, and elastic component of shear stresses with increasing $V_{0}$. Initially, the movement of the block to be sheared $\left(V_{0}=V_{01}\right)$ results in increasing friction force at small $f$. When the elastic shear stresses reach their critical value, the lubricant shear melting occurs following the mechanism of first-order phase transition. Here, the parameter $f$ increases stepwise and the elastic stresses become zero. After that, the lubricant starts solidifying again since the relative shear velocity of the friction surfaces decreases (see Fig. 7). When the 


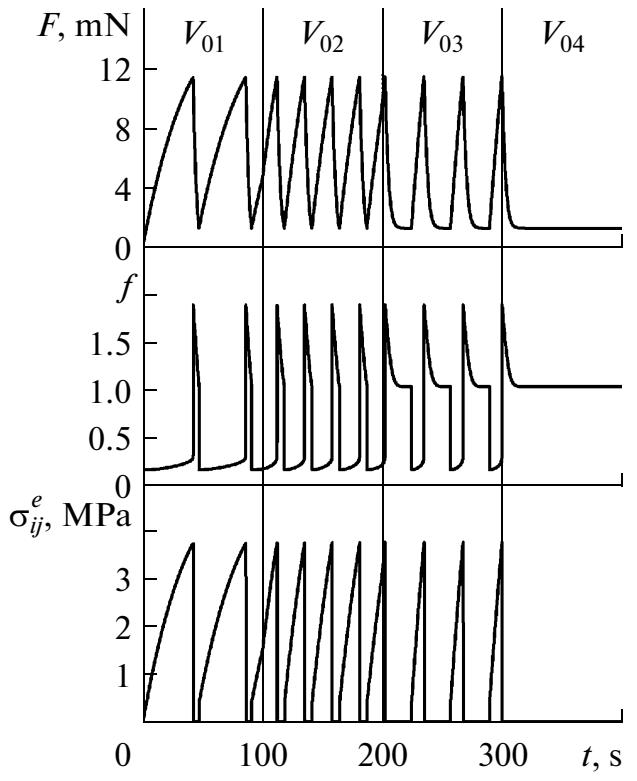

Fig. 8. Time dependences of friction force $F$, excess volume $f$, and elastic component of shear stresses $\sigma_{i j}^{e}$ at parameters of Fig. 7, normal stresses $n=-30 \times 10^{5} \mathrm{~Pa}$, and shear rates $V_{01}=900 \mathrm{~nm} / \mathrm{s} ; V_{02}=1600 \mathrm{~nm} / \mathrm{s} ; V_{03}=2365 \mathrm{~nm} / \mathrm{s}$; and $V_{04}=2370 \mathrm{~nm} / \mathrm{s}$.

lubricant solidifies completely, elastic stresses appear in it and their further growth again leads to $f$ increasing up to the critical value necessary for melting, upon which the process is repeated again. As a result, the periodic stick-slip regime of melting/solidification is

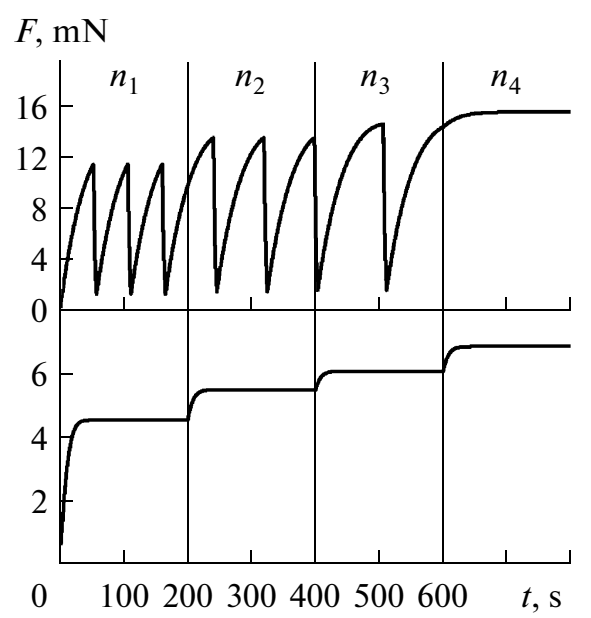

Fig. 9. Time dependence of friction force at parameters of Fig. 7, shear velocity $V_{0}=800 \mathrm{~nm} / \mathrm{s}$, and external normal load $n_{1}=-7 \times 10^{5} \mathrm{~Pa} ; n_{2}=-40 \times 10^{5} \mathrm{~Pa} ; n_{3}=-50 \times 10^{5} \mathrm{~Pa}$; $n_{4}=-60 \times 10^{5} \mathrm{~Pa}$. The upper and lower parts of the figure correspond to temperatures $T=235 \mathrm{~K}$ and $T=350 \mathrm{~K}$.

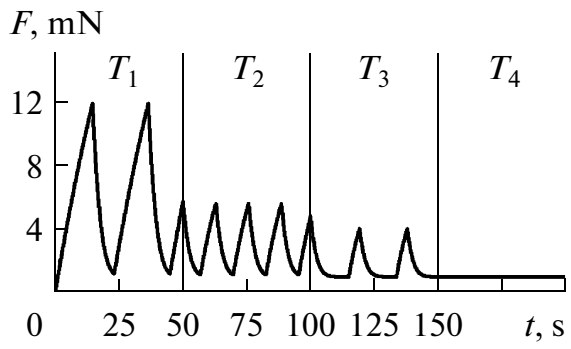

Fig. 10. Time dependence of friction force at parameters of Fig. 7, velocity $V_{0}=2000 \mathrm{~nm} / \mathrm{s}$ and lubricant temperature $T_{1}=230 \mathrm{~K} ; T_{2}=300 \mathrm{~K} ; T_{3}=438 \mathrm{~K} ; T_{4}=439 \mathrm{~K}$.

established. When the velocity is increased up to $V_{0}=$ $V_{02}$ the frequency of the stick-slip peaks increases since at this velocity the critical stress is reached more quickly. Correspondingly, the lubricant is melted faster and during the same period of time it manages to make more melting/solidification transitions. At even larger velocity $V_{0}=V_{03}$, the frequency of stick-slip peaks again decreases due to the appearance of kinetic regions $F=$ const on the dependence $F(t)$. One should note that in melting under these conditions, the excess volume $f$ first increases sharply owing to the fast growth of the shear velocity $V$ of the upper rubbing block and the stationary kinetic region corresponds to a lower excess volume $f$ that is established after the initial slip of the rubbing surface due to the release of some part of the mechanical potential energy of the compressed spring. With further growth of $V_{0}=V_{04}$, the stick-slip regime disappears and the kinetic friction regime of the liquid lubricant is set. Thus, with increasing velocity the frequency of stick-slip peaks first increases and then decreases due to the appearance of long kinetic spots; when the critical velocity $V_{0}$ is exceeded, the stick-slip regime disappears. The behavior described agrees well with the experimental results of work [3].

The influence of external pressure applied normally to the friction surfaces on lubricant melting is also often studied experimentally [3, 41]. These experiments show that pressure has an effect on the parameters of the tribological system in a nontrivial way, e.g., in the lubricant made up of a chain molecules of hexadecane the critical shear velocity decreases with increasing pressure and in the spherical molecules of OMCTS it increases [3]. Pressure also has an effect on the frequency and amplitude of stick-slip transitions [3]. Within the framework of the model proposed according to Eq. (11), an increase in the load on the friction surface leads to decreasing excess volume, which facilitates lubricant solidification.

The time dependence of the friction force at different pressures which tend to confine the friction surfaces is shown in Fig. 9. At temperatures lower than the critical value (the upper part of the figure), the stick-slip regime is established, the amplitude of stick- 
slip transitions increasing and their frequency decreasing with increasing pressure. Under a pressure corresponding to normal stresses $n=n_{4}$, the stick-slip regime is not established. However, in this case lubricant solidification occurs due to wall confinement rather than the kinetic regime conforming to lubricant melting. Due to this, the lubricant cannot melt and a large friction force $F$ is established with the solid lubricant since the wall confinement facilitates the occurrence of long-order atom alteration in the lubricant. On the lower part of the figure one can see the dependence at higher $T$. Here, the kinetic friction regime corresponds to a small friction force. With further increase in pressure one should expect transition to the stick-slip regime and with further increase in load we would expect full lubricant solidification, as is shown in the upper part of the figure at $n=n_{4}$. Thus, three friction regimes have been identified: (1) the kinetic regime within which lubricant has solid-like structure; (2) the stick-slip regime corresponding to periodic melting/solidification; and (3) dry friction characterized by a large friction force and solid structure of the lubricant. Similar regimes are also found within the framework of the stochastic model [19].

In line with Fig. 10, the elevation of lubricant temperature results in a lower amplitude of friction force oscillations and variations in the frequency of phase transitions. At $T=T_{4}$, the sliding regime is characterized by a constant kinetic friction force and shear velocity of the rubbing block. Thus, temperature elevation facilitates lubricant melting. The authors do not have data from experiments studying the temperature effects; therefore, the dependence obtained is predictive in nature.

\section{CONCLUSIONS}

The model proposed allows description of the effects observed upon melting of an ultrathin lubricant film under boundary friction conditions. Thermodynamic and shear melting have been considered. At high lubricant temperatures, shear melting occurs at lower shear velocities and with further temperature elevation the lubricant is in the liquid state even at zero shear velocity.

The phase diagram has been constructed for the case of a constant shear velocity of the upper rubbing block. We have also considered the dependences of the friction force on shear velocity, temperature, and pressure applied to the rubbing surfaces at a constant shear velocity using a real tribological system as an example. The stick-slip friction regime is described, which arises due to the oscillating nature of the friction force. The results obtained agree well with the experimental findings of other researchers.

The work was supported by the State Foundation for Basic Research of Ukraine and the Russian Foundation for Basic Research (grant $\Phi 28 / 443-2009$ ).

\section{APPENDIX: DETERMINATION \\ OF STRAIN TENSOR INVARIANTS}

For an isotropic body the following equality is valid [43]:

$$
\sigma_{i j}=\lambda \varepsilon_{i j} \delta_{i j}+2 \mu \varepsilon_{i j} .
$$

We choose the main axes of strain as coordinate axes; then the strain tensor $\varepsilon_{i j}$ has only diagonal components. Let us consider the flat deformed state of the lubricant in which $\varepsilon_{22}=0$.

The diagonal components of the stress tensor in this system of coordinates take the form

$$
\begin{gathered}
\sigma_{1}=\lambda\left(\varepsilon_{11}+\varepsilon_{33}\right)+2 \mu \varepsilon_{11} ; \\
\sigma_{2}=\lambda\left(\varepsilon_{11}+\varepsilon_{33}\right) ; \\
\sigma_{3}=\lambda\left(\varepsilon_{11}+\varepsilon_{33}\right)+2 \mu \varepsilon_{33} .
\end{gathered}
$$

Along the lubricant boundary the tangential stress operates [42]

$$
\tau_{2}=\frac{\sigma_{3}-\sigma_{1}}{2}=\mu\left(\varepsilon_{33}-\varepsilon_{11}\right),
$$

and the stress being normal to the lubricant boundary is [42]

$$
n_{2}=\frac{\sigma_{3}+\sigma_{1}}{2}=(\lambda+\mu)\left(\varepsilon_{11}+\varepsilon_{33}\right) .
$$

Then we have from (A.5) and (A.6)

$$
\begin{aligned}
& \varepsilon_{11}=\frac{1}{2}\left(\frac{n_{2}}{\lambda+\mu}-\frac{\tau_{2}}{\mu}\right) ; \\
& \varepsilon_{33}=\frac{1}{2}\left(\frac{n_{2}}{\lambda+\mu}+\frac{\tau_{2}}{\mu}\right) .
\end{aligned}
$$

Correspondingly, first two invariants (8) and (9) of the strain tensor will be determined as

$$
\begin{aligned}
\varepsilon_{i i}= & \varepsilon_{11}+\varepsilon_{22}+\varepsilon_{33}=\frac{n_{2}}{\lambda+\mu} ; \\
\varepsilon_{i j} \varepsilon_{j i} & =\left(\varepsilon_{11}\right)^{2}+\left(\varepsilon_{22}\right)^{2}+\left(\varepsilon_{33}\right)^{2} \\
& =\frac{1}{2}\left[\left(\frac{\tau_{2}}{\mu}\right)^{2}+\left(\varepsilon_{i i}\right)^{2}\right]
\end{aligned}
$$

\section{DESIGNATIONS}

$f$-excess volume (disorder parameter); $\Phi$-density of free energy; $\Phi_{0}, c, \phi_{0}, \phi_{1}, \phi_{2}, \phi_{3}$ - parameters of expansion; $\varepsilon_{i j}^{e}$-tensor of elastic strains; $T$-lubricant temperature; $\Phi_{0}^{*}, \lambda, \mu$-parameters of expansion; $\left(\varepsilon_{i i}^{e}\right)^{2}$-squared first invariant of strain tensor; $\left(\varepsilon_{i j}^{e}\right)^{2}=$ $\left(\varepsilon_{1}^{e}\right)^{2}+\left(\varepsilon_{2}^{e}\right)^{2}+\left(\varepsilon_{3}^{e}\right)^{2}-$ second invariant of strain ten- 
sor; $\phi_{0}^{*}, \bar{\lambda}, \bar{\mu}, \alpha$-expansion parameters; $\varepsilon_{1}^{e}, \varepsilon_{2}^{e}$, $\varepsilon_{3}^{e}$-diagonal components of strain tensor; $\left(\varepsilon_{l l}^{e}\right)^{2}-$ square of the trace of strain tensor; $I_{2}=\varepsilon_{1}^{e} \varepsilon_{2}^{e}+\varepsilon_{1}^{e} \varepsilon_{3}^{e}+$ $\varepsilon_{2}^{e} \varepsilon_{3}^{e}$-second invariant of strain tensor; $\sigma_{i j}^{e}$-tensor of elastic stresses; $\delta_{i j}$-Kronecker delta-symbol; $\mu_{e f f}$, $\lambda_{\text {eff }}$-effective elastic constants (Lame constants); $n$-normal component of stresses applied to friction surfaces; $\tau$-tangential component of stresses; $\tau_{f}$-time of excess volume relaxation; $V_{i j}$-tensor of shear velocities of friction surfaces; $\varepsilon_{i j}^{p l}$-tensor of plastic strains; $\tau_{\varepsilon}$ Maxwell time of stress relaxation; $\varepsilon_{i j}$-tensor of full strains; $h$-lubricant film thickness; $\varepsilon_{i j 0}^{e}$-stationary tensor of elastic strains; $A$-contact area of friction surfaces; $L$-external pressure applied to friction surfaces; $F$-friction force; $T_{c 0}$ - critical temperature of lubricant melting; $T_{c}^{0}$ — critical temperature of lubricant solidification; $V_{c 0}$-critical shear velocity at which lubricant melts; $V_{c}^{0}$ —critical shear velocity at which lubricant solidifies; $\Delta T=T_{c 0}-T_{c}^{0}$ —width of hysteresis by temperature; $\sigma_{i j}^{\text {visc }}$-tensor of viscous stresses; $\sigma_{i j}$ - tensor of full stresses; $F_{i j}$-tensor of friction forces; $\eta_{\text {eff }}$-effective viscosity; $\gamma$-phenomenological coefficient; $K$-coefficient of spring rigidity; $M$-mass of upper rubbing block; $V_{0}$ - movement velocity of spring end; $V$-movement velocity of rubbing block; $X$ - coordinate of upper rubbing block; $t$ - time; $V_{01}, V_{02}, V_{03}$, $V_{04}$-fixed velocities; $n_{1}, n_{2}, n_{3}, n_{4}$-fixed normal stresses; $T_{1}, T_{2}, T_{3}, T_{4}$-fixed lubricant temperatures.

\section{REFERENCES}

1. Persson, B.N.J., Sliding Friction. Physical Principles and Applications, Berlin: Springer, 2000.

2. Persson, B.N.J., Albohr, O., Tartaglino, U., et al., On the Nature of Surface Roughness with Application to Contact Mechanics, Sealing, Rubber Friction and Adhesion, J. Phys.: Condens. Matter, 2005, vol. 17, pp. R1-R62.

3. Yoshizawa, H., Chen, Y.-L., and Israelachvili, J., Fundamental Mechanisms of Interfacial Friction. 1. Relation between Adhesion and Friction, J. Phys. Chem., 1993, vol. 97, pp. 4128-4140; Yoshizawa H. and Israelachvili J. Fundamental Mechanisms of Interfacial Friction. 2. Stick-Slip Friction of Spherical and Chain Molecules, J. Phys. Chem., 1993, vol. 97, pp. 1130011313.

4. Smith, E.D., Robbins, M.O., and Cieplak, M., Friction on Adsorbed Monolayers, Phys. Rev. B: Condens. Matter, 1996, vol. 54, pp. 8252-8260.

5. Israelachvili, J.N. and Adams, G.E., Measurement of Forces between Two Mica Surfaces in Aqueous Electrolyte Solutions in the Range 0-100 Nm, J. Chem. Soc.,
Faraday Trans. 1: Phys. Chem. Cond. Phases, 1978, vol. 74, pp. 975-1001.

6. Pashley, R.M., Hydration Forces between Mica Surfaces in Aqueous Electrolyte Solutions, J. Colloid Interface Sci., 1981, vol. 80, pp. 153-162.

7. Pashley, R.M., DLVO and Hydration Forces between Mica Surfaces in $\mathrm{Li}^{+}, \mathrm{Na}^{+}, \mathrm{K}^{+}$, and $\mathrm{Cs}^{+}$Electrolyte Solutions: A Correlation of Double-Layer and Hydration Forces with Surface Cation Exchange Properties, J. Colloid Interface Sci., 1981, vol. 83, pp. 531-546.

8. Horn, R.G., Smith, D.T., and Haller, W., Surface Forces and Viscosity of Water Measured between Silica Sheets, Chem. Phys. Lett., 1989, vol. 162, pp. 404-408.

9. Horn, R.G., Clarke, D.R., and Clarkson, M.T., Direct Measurement of Surface Forces between Sapphire Crystals in Aqueous Solutions, J. Mater. Res., 1988, vol. 3, pp. 413-416.

10. Ploehn, H.J. and Russel, W.B., Interactions between Colloidal Particles and Soluble Polymer, Adv. Chem. Eng., 1990, vol. 15, pp. 137-228.

11. Israelachvili, J.N., Intermolecular and Surface Forces: with Applications to Colloidal and Biological Systems, New York: Academic Press, 1991.

12. Israelachvili, J.N. and McGuiggan, P.M., Forces between Surfaces in Liquids, Science, 1988, vol. 241, pp. 795-800.

13. Lee, C.S. and Belfort, G., Changing Activity of Ribonuclease A during Adsorption: A Molecular Explanation, Proc. NAS USA, 1989, vol. 86, pp. 8392-8396.

14. Parker, J.L. and Christenson, H.K., Measurements of the Forces between a Metal Surface and Mica across Liquids, J. Chem. Phys., 1988, vol. 88, pp. 8013-8014.

15. Popov, V.L., Thermodynamics and Kinetics of ShearInduced Melting of a Thin Layer of Lubricant Confined between Solids, Zh. Tekhn. Fiz., 2001, vol. 71, no. 5, pp. 100-110 [Techn. Phys. (Engl. Transl.), vol. 46, no. 5, pp. 605-615].

16. Carlson, J.M. and Batista, A.A., Constitutive Relation for the Friction between Lubricated Surfaces, Phys. Rev. E, 1996, vol. 53, pp. 4153-4165.

17. Khomenko, A.V. and Yushchenko, O.V., Solid-Liquid Transition of Ultrathin Lubricant Film, Phys. Rev. E, 2003, vol. 68, p. 036110.

18. Khomenko, A.V. and Lyashenko, I.A., Temperature Dependence Effect of Viscosity on Ultrathin Lubricant Film Melting, Cond. Matt. Phys., 2006, vol. 9, pp. 695702.

19. Filippov, A.E., Klafter, J., and Urbakh, M., Friction through Dynamical Formation and Rupture of Molecular Bonds, Phys. Rev. Lett., 2004, vol. 92, p. 135503.

20. Tshiprut, Z., Filippov, A.E., and Urbakh, M., Tuning Diffusion and Friction in Microscopic Contacts by Mechanical Excitations, Phys. Rev. Lett., 2005, vol. 92, p. 016101.

21. Braun, O.M. and Naumovets, A.G., Nanotribology: Microscopic Mechanisms of Friction, Surf. Sci. Rep., 2006, vol. 60, pp. 79-158.

22. Khomenko, A.V. and Prodanov, N.V., Molecular Dynamics Simulations of Ultrathin Water Film Confined between Flat Diamond Plates, Cond. Matt. Phys., 2008, vol. 11, pp. 615-626. 
23. Brener, E.A. and Marchenko, V.I., Frictional Shear Cracks, Pis'ma Zh. Eksp. Teor. Fiz., 2002, vol. 76, pp. 246-249 [JETP Lett. (Engl. Transl.), vol. 76, no. 4, pp. 211-214.

24. Khomenko, A.V. and Lyashenko, I.A., Stochastic Theory of Ultrathin Lubricant Film Melting in the StickSlip Regime, Zh. Tekhn. Fiz., 2005, vol. 75, no. 11, pp. 17-25 [Techn. Phys. (Engl. Transl.), vol. 50, no. 11, pp. 1408-1416].

25. Khomenko, A.V. and Lyashenko, I.A., Melting of Ultrathin Lubricant Film due to Dissipative Heating of Friction Surfaces, Zh. Tekhn. Fiz., 2007, vol. 77, no. 9, pp. 138-141 [Techn. Phys. (Engl. Transl.), vol. 52, no. 9, pp. 1239-1243].

26. Khomenko, A.V., Lyashenko, I.A., and Borisyuk, V.M., Self-Similar Phase Dynamics of Boundary Friction, Ukr. Fiz. Zh., 2009, vol. 54, no. 11, pp. 1142-1151 [Ukrainian Journal of Physics (Engl. Transl.), vol. 54, pp. 1139-1148].

27. Khomenko, A.V., Lyashenko, I.A., and Borisyuk, V.N., Multifractal Analysis of Stress Time Series during Ultrathin Lubricant Film Melting, Fluct. Noise Lett., 2010, vol. 9, pp. 19-35.

28. Khomenko, A.V. and Lyashenko, I.A., Phase Dynamics and Kinetics of Thin Lubricant Film Driven by Correlated Temperature Fluctuations, Fluct. Noise Lett., 2007, vol. 7, pp. L111-L133.

29. Demirel, A.L. and Granick, S., Transition from Static to Kinetic Friction in a Model Lubricating System, J. Chem. Phys., 1998, vol. 109, pp. 6889-6897.

30. Reiter, G., Demirel, A.L., Peanasky, J., et al., Stick to Slip Transition and Adhesion of Lubricated Surfaces in Moving Contact, J. Chem. Phys., 1994, vol. 101, pp. 2606-2615.

31. Israelachvili, J., Adhesion Forces between Surfaces in Liquids and Condensable Vapors, Surf. Sci. Rep., 1992, vol. 14, pp. 109-159.

32. Khomenko, A.V. and Lyashenko, I.A., Hysteresis Phenomena during Melting of an Ultrathin Lubricant Film, Fiz. Tver. Tela, 2007, vol. 49, no. 5, pp. 886-890 [Phys. Solid State (Engl. Transl.), vol. 49, no. 5, pp. 936-940].

33. Khomenko, A.V. and Lyashenko, I.A., Hysteresis Phenomena at Ultrathin Lubricant Film Melting in the
Case of First-Order Phase Transition, Phys. Lett. A, 2007, vol. 366, pp. 165-173.

34. Khomenko, A.V. and Lyashenko, I.A., Phase Dynamics of Thin Film between Solid Surfaces at the Strain Defect of Shear Modulus, Zh. Fiz. Dosl., 2007, vol. 11, pp. 268-278 [J. Phys. Studies (Engl. Transl.), vol. 11, pp. 268-278 (in Ukrainian)].

35. Khomenko, A.V. and Lyashenko, I.A., Periodic Intermittent Regime of a Boundary Flow, Zh. Tekhn. Fiz., 2010, vol. 80, no. 1, pp. 27-33 [Techn. Phys. (Engl. Transl.), vol. 55, no. 1, pp. 26-32].

36. Metlov, L.S., Thermodynamics of Severe Plastic Strains, Metallofiz. Noveish. Tekhnol., 2007, vol. 29, pp. 335-345.

37. Metlov, L.S., Thermodynamics of Nonequilibrium Processes in Application to Severe Plastic Strain, Izv. RAN. Ser. Fiz., 2008, vol. 72, no. 9, pp. 1353-1357 [Bull. Russ. Acad. Sci.: Phys. (Engl. Transl.), vol. 72, no. 9, pp. 1283-1287].

38. Khomenko, A.V., Lyashenko, I.A., and Metlov, L.S., Phase Dynamics and Kinetics of Intensive Plastic Strain, Metall. Noveish. Tekhnol., 2008, vol. 30, pp. 859-872.

39. Metlov, L.S., Formation of Internal Structure of Solids under Severe Load, Phys. Rev. E, 2010, vol. 81, p. 051121 .

40. Thompson, P.A., Grest, G.S., and Robbins, M.O., Phase Transitions and Universal Dynamics in Confined Films, Phys. Rev. Lett., 1992, vol. 68, pp. 3448-3451.

41. Gee, M.L., McGuiggan, P.M., and Israelachvili, J.N., Liquid to Solidlike Transitions of Molecularly Thin Films under Shear, J. Chem. Phys., 1990, vol. 93, pp.1895-1906.

42. Kachanov, L.M., Osnovy teorii plastichnosti (Foundations of the Theory of Plasticity), Moscow: Nauka, 1969; Amsterdam: North-Holland Publ. Com., 1971.

43. Landau, L.D. and Lifshits, E.M., Teoriya uprugosti (Course of theoretical physics, vol.7: Theory of elasticity), Moscow: Nauka, 2007. 3rd ed. New York: Pergamon Press. 1986.

44. Luengo, G., Israelachvili, J., and Granick, S., Generalized Effects in Confined Fluids: New Friction Map for Boundary Lubrication, Wear, 1996, vol. 200, pp. 328-335. 